\section{Neck Dissection bei Kopf-Hals-Tumoren: Zahl der Lymphknoten entscheidet}

\begin{abstract}
Prospektiv wurde untersucht, wie sich die Zahl der entfernten Lymphknoten bei der Neck-Dissection wegen eines Plattenepithelkarzinoms im Kopf-HalsBereich auf Rezidivrisiko und Gesamtüberleben auswirkt.
\end{abstract}

$\mathrm{P}_{\mathrm{z}}^{\mathrm{ro}}$ rospektive Daten zur Korrelation der Zahl entfernter Lymphknoten bei Neck-Dissection und der Prognose lagen bislang nicht vor. In der aktuellen Analyse wurde bei insgesamt 572 Patienten aus 2 randomisierten Studien der Radiation Therapy Oncology Group (RTOG) die Zahl der bei Neck-Dissection entfernten Lymphknoten ermittelt und zum Überleben in Beziehung gesetzt; zum einen aus der Phase-III-Studie RTOG 9501, in der die Radiotherapie (RT) allein versus RT mit begleitender Cisplatin-Chemotherapie verglichen wurde und zum anderen aus der PhaseII-Studie RTOG 0234. In dieser wurde die RT mit begleitendem Cisplatin/Cetuximab bzw. Docetaxel/Cetuximab versus den Chemoradiotherapie-Arm der RTOG 9501-Studie getestet.

Das mediane Follow-up betrug 8 Jahre. Bei $98 \%$ der Patienten fand sich ein Lymphknotenbefall (pathologisch $\mathrm{N}+$ ). Entfernt wurden median 24 Lymphknoten der linken und 25 der rechten Seite. Wurden weniger als 18 Lymphknoten gefunden und entfernt, war das Gesamtüberleben (OS) in der multivariaten Analyse signifikant schlechter als bei $\geq 18$ Lymphknoten (Hazard Ratio [HR] 1,38; $\mathrm{p}=0,007)$. Der Cut-off-Wert „18 Lymphknoten“ zeigte im Modell verglichen mit allen anderen Werten maximalen Effekt (größte HR). Die Unterschiede im OS zwischen $<18$ und $\geq 18$ entfernten Lymphknoten kamen durch häufige- re lokoregionäre Rezidive zustande (HR $1,46 ; \mathrm{p}=0,04)$. Patienten, bei denen $<18$ Lymphknoten entfernt wurden, entwickelten aber nicht häufiger distale Metastasen als die übrigen (HR 1,08; $\mathrm{p}=0,65$ ).

Der in der RTOG 0234-Studie zusätzlich ermittelte p16-Status (von 130 Patienten $43,8 \%$ p16-positiv) hatte keinen Einfluss. Die mediane Zahl der resezierten Lymphknoten betrug 27 bei den p16positiven Patienten und 23 bei den p16negativen $(\mathrm{p}=0,14)$.

Fazit: Die Identifizierung und Entfernung von $\geq 18$ Lymphknoten bei der Neck-Dissection wegen eines Plattenepithelkarzinoms im Kopf-Hals-Bereich war mit einem besseren OS und einer niedrigeren Rate an lokoregionären Rezidiven verbunden. Dies galt für p16-positive und p16-negative Tumoren gleichermaßen. Kathrin von Kieseritzky

Divi V et al. Establishing Quality Indicators for Neck Dissection: Correlating the Number of Lymph Nodes With Oncologic Outcomes (NRG Oncology RTOG 9501 and RTOG 0234). Cancer. $2016 \mathrm{Jul}$ 15. [Epub ahead of print].

\title{
Rezidiv bei Kopf-Hals-Tumoren: SBRT mit Langzeitschäden?
}

Bei lokal rezidivierten, zuvor bestrahlten Kopf-Hals-Tumoren, die für eine Salvage-Operation ungeeignet sind, hat sich die Körperstereotaxie (SBRT) als effizient erwiesen. Doch wie steht es mit der Langzeittoxizität?

A uch die SBRT kann - obwohl gegenüber konventioneller Re-Bestrahlung und systemischer Therapie verträglicher - schwere akute und Spätkomplikationen verursachen. In einer retrospektiven Studie wurden die Daten von 291 Patienten ausgewertet, die zwischen April 2002 und März 2013 am University of Pittsburgh Cancer Institute, PA/ USA, wegen eines rezidivierten KopfHals-Tumors eine SBRT (mit kurativer Intention oder palliativ) erhalten hatten. Bei allen war der Krebs zuvor bestrahlt worden, mit oder ohne Operation oder Chemotherapie. Ausgeschlossen wurden Patienten mit weniger als 3 Monaten Follow-up $(\mathrm{n}=64)$.

Die mediane Zeit bis zu Tod oder letztem klinischem Follow-up betrug 9,8
Monate in der Gesamtkohorte und 53,1 Monate unter den Überlebenden. Insgesamt verstarben im gesamten Zeitraum 264 Patienten (90,7\%). Die 1-, 3-, 5- und 10-Jahres-Gesamtüberlebensraten lagen bei $41,4 \%, 16,6 \%, 10,8 \%$ und $3,6 \%$.

43 von 227 Patienten (18,9\%) erlebten späte Toxizitäten vom Grad $\geq 3$. Grad3-Nebenwirkungen waren Dysphagie $(8,8 \%)$, Osteonekrose $(2,6 \%)$, LarynxÖdem $(0,9 \%)$, Trismus $(0,9 \%)$ und tracheal-ösophageale Fisteln (0,9\%), Grad4-Nebenwirkungen Larynx-Stenose/ Ödem (0,9\%), Temporallappennekrose $(0,4 \%)$, Blutung eines Pseudoaneurysmas der Karotis interna nach Strahlennekrose $(0,4 \%)$ und tracheal-ösophageale Fisteln (0,4\%). Grad-5-Nebenwirkungen waren Karotis Blow-out-Syn- drom bei Fehlen eines Rezidivs (1,3\%), Dysphagie (0,9\%), Larynx-Ödem $(0,4 \%)$ und Mukosablutung (0,4\%).

Rezidive von Tumoren des Larynx-/ Hypopharynx waren mit signifikant mehr schweren Spätkomplikationen assoziiert als Rezidive anderer Lokalisationen (50 vs. $6-20 \%$; $<<0,05)$.

Fazit: Die Salvage-SBRT ist für die meisten Patienten mit zuvor bestrahltem Kopf-Hals-Tumor eine sichere und effiziente Option. Insbesondere Patienten mit Larynx- oder Hypopharynxkarzinom erleben aber nicht selten schwere Spättoxizitäten der Behandlung, weshalb bei der Auswahl der Patienten für diese Therapie besondere Sorgfalt geboten ist.

Kathrin von Kieseritzky

Ling DC et al. Risk of Severe Toxicity According to Site of Recurrence in Patients Treated With Stereotactic Body Radiation Therapy for Recurrent Head and Neck Cancer. Int J Radiat Oncol Biol Phys. 2016;95(3):973-80. 